\title{
APPLICATION OF YIELD LOCI CALCULATED FROM TEXTURE DATA
}

\author{
P. VAN HOUTTE, K. MOLS, A. VAN BAEL and E. AERNOUDT \\ Department of Metallurgy and Materials Engineering, Katholieke Universiteit \\ Leuven, de Croylaan 2, B-3030 Leuven, Belgium
}

(Received February 2, 1989)

\begin{abstract}
The concept of the yield locus as a means of representing the plastic anisotropy of a textured material is remembered. It is shown how such yield loci can be used in a very general way, i.e. in full six-dimensional stress space. As an example of how such yield loci can actually be obtained, the series expansion method based on Taylor factors is explained. It is finally shown that these six-dimensional yield loci can be approximated by analytical expressions and under such form brought into finite element calculations of elasto-plastic forming processes.
\end{abstract}

KEY WORDS Yield locus, six-dimensional, Taylor model, series expansion, analytical expression.

\section{INTRODUCTION}

\subsection{The Concept of a Yield Locus}

Yield loci are tools used in the analysis of plastic forming problems. They provide a means of knowing whether a uniaxial or multiaxial stress state can cause plastic deformation in a given material. Moreover, they can be used to find the plastic strain rate tensor that corresponds to a plastic stress by applying Hill's Maximum Work criterion (Hill, 1950).

A stress is in general described by six independent components: $S_{11}, S_{22}, S_{33}, S_{23}=S_{32}, S_{31}=S_{13}$ and $S_{12}=S_{21}$. A condition expressing that a given stress state $S_{i j}$ causes plastic flow in a given material generally takes the form:

$$
F\left(S_{i j}\right)=0
$$

For incompressible materials, the yield condition does not depend on the hydrostatic component of the stress, but only on the deviatoric stress $S^{\prime}$. It is then often convenient to use expressions for the yield condition in which the $S_{i j}$ have been replaced by $S_{i j}^{\prime}$. Moreover, there are only five independent deviatoric stress components in such a case, which certainly is important for the analysis (Van Houtte, 1987, 1988; Lequeu et al., 1987). For reasons of clarity, we will not emphasize this aspect in the present paper.

Note that for a given material, the actual form of expression (1) will depend on the choice of the reference system used in physical space. Such condition can be represented geometrically in a six dimensional space, of which each axis corresponds to one of the independent stress components. Such representation is called a yield surface or yield locus. It evidently is impossible to visualize such six 
dimensional yield locus in a single drawing. It is customary to represent plane sections of the yield locus. Many different plane sections can be made; see for example the cases discussed by Van Houtte (1987). Such plane section can generally be described as being an $x, y$ plot in which each combination of $x$ and $y$ stands for the following stress state:

$$
\mathbf{S}=x \mathbf{S}_{x}+y \mathbf{S}_{y}
$$

The "basis vectors" $\mathbf{S}_{x}$ and $\mathbf{S}_{\boldsymbol{y}}$ are often very simple: $\mathbf{S}_{x}$ may for example represent a uniaxial stress $S_{11}$ and $\mathbf{S}_{y}$ a uniaxial stress $S_{22}$ (Figure 1). More complex examples have been given by Van Houtte (1987).

A first application of a yield locus (assuming that it is known) is to answer the question: at what stress level will plastic yielding begin for a given stress mode $\mathbf{U}$ ? (Figure 1, see also next section).

A second application is to answer the question: what do we know about the (plastic) strain rate tensor when we know the plastic stress? Or conversely, what is the plastic stress that is associated to a given plastic strain rate $\dot{\mathbf{E}}$ ? The answer to these questions is given by the Maximum Work Criterion (Hill, 1950). The following equation can be derived from it for incompressible materials with "smooth" yield loci (without vertices):

$$
\dot{E}_{i j}=\lambda \frac{\partial F\left(S_{k l}^{\prime}\right)}{\partial S_{i j}^{\prime}}
$$

in which $S_{i j}^{\prime}$ are the components of the deviatoric stress tensor. $\lambda$ must be non-negative but is not fixed otherwise. Only models that asume strain rate sensitivity can connect a plastic stress $\mathbf{S}$ to a particular strain rate $\dot{\mathbf{E}}$.

\subsection{The Concept of a Stress Mode}

The concept of "stress mode" has been introduced by Aernoudt, Gil Sevillano and Van Houtte (1987). It merely is a fixed direction in stress space, described by

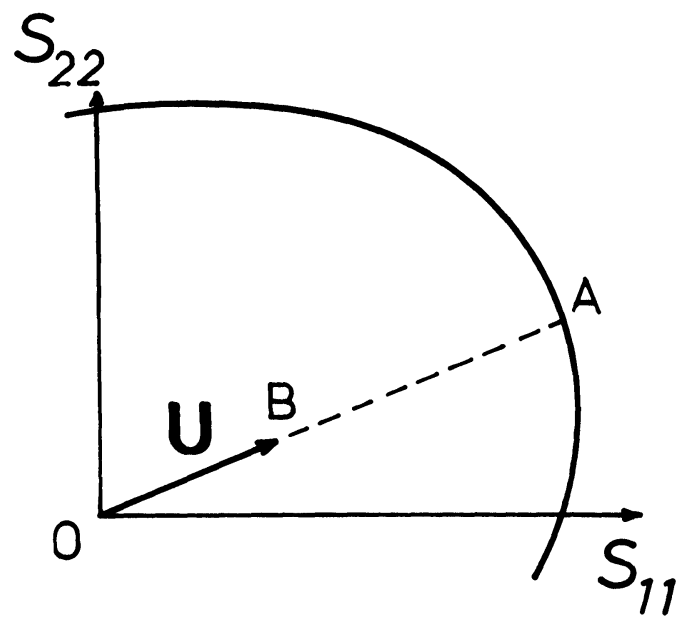

Figure 1 An example of a two-dimensional section of a yield locus. $\mathbf{U}$ defines a direction in such diagram: a "Stress mode." 


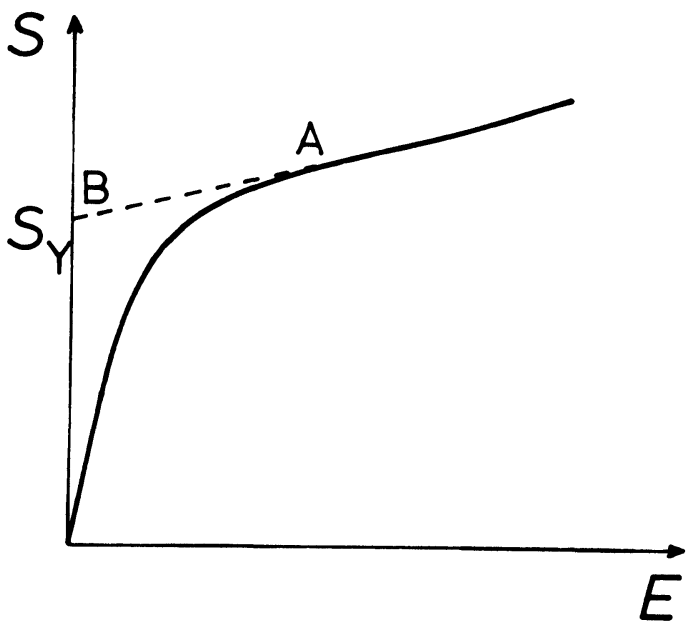

Figure 2 Typical stress-strain curve. After an elastic-plastic transient, a linear part is often reached (A). This can be back-extrapolated leading to the "back-extrapolated yield stress" $S_{Y}$.

a "vector" $\mathbf{U}$ which in reality has the nature of a tensor. $\dagger$ In Figure 1 , the plastic stress state in the direction defined by $\mathbf{U}$ is represented by the point $A$. The stress "Vector" pointing to $A$ is given by

$$
\mathbf{S}_{Y}=S_{Y} \mathbf{U}
$$

in which the scalar $S_{Y}$ is the ratio $O A / O B$. It has been convened that $S_{Y}$ has the dimensions of a stress (force/surface) while $\mathbf{U}$ is dimensionless.

\subsection{Back-Extrapolated Yield Stresses}

There may be a problem in defining the value of $S_{Y}$. It will indeed depend on the value of the strain at which it is measured. Figure 2 shows a typical stress-strain curve. Assume that $S$ is the level of the stress in a uniaxial tension test, and that $E$ stands for the true strain in the tensile direction. One may conventionally define a "yield stress" at an offset, i.e. the stress for which the non-proportional part of the strain reaches a certain value, such as $0.01 \%$ or $0.2 \%$. The present authors however prefer to use a type of "yield stress" that is relevant for the stresses that occur in industrial forming operations, for which the total strains nearly always exceed $5 \%$. This means that it is not necessary to consider the elastic-plastic transition region. On the other hand, the stress level will be affected by strain hardening at strain levels of 5\% and more. The "back extrapolated yield stress" is often used to overcome these problems. The true stress-strain curve sometimes reaches a linear part after the elastic-plastic transition region (A in Figure 2). This linear part is back-extrapolated to the $S$-axis which then gives the "back-extrapolated yield stress" $S_{Y}$ (at B in Figure 2).

$\dagger$ Rigorous methods for converting deviatoric stress tensors or strain rate tensors into vectors have been proposed by Lequeu, Gilormini, Montheillet, Bacroix and Jonas (1987) and by Van Houtte (1988). 
Aernoudt et al. (1987) proposed a method to define a back extrapolated stress for multiaxial tests as well. $S$ is no longer the uniaxial stress in tensile test, but is a stress level such that the stress tensor is given by:

$$
\mathbf{S}=S \mathbf{U}
$$

in which $\mathbf{U}$ is the stress mode. $\mathbf{U}$ is supposed to be constant during the test. It may represent a pure shear stress, a biaxial tensile stress etc. Let the tensor $\dot{\mathbf{E}}$ represent the strain rate at a given moment $t$ during the test. A scalar $\dot{E}$ may be defined as follows:

$$
\dot{E}=\mathbf{U}: \dot{\mathbf{E}}
$$

$\dot{E}$ has the dimension (time) ${ }^{-1}$ since $\mathbf{U}$ is dimensionless. A measure for the total strain is then defined as follows:

$$
E=\int_{0}^{t} \dot{E} \mathrm{~d} t
$$

Note that these definitions are such that $P$, the power dissipation by unit volume can be calculated from the scalars $S$ and $\dot{E}$. It is indeed seen that

$$
P=\mathbf{S}: \dot{\mathbf{E}}=S \mathbf{U}: \dot{\mathbf{E}}=S \dot{E}
$$

Scalars $S$ and $E$ have now been defined for multiaxial tests, which then enables to make a plot such as Figure 2 for such tests as well. A back-extrapolated plastic stress level $S_{Y}$ can hence be defined, as well as the back-extrapolated yield stress tensor $S_{Y}$ (Eq. (4)).

These yield stresses also depend on temperature and on strain rate. These effects will not be considered further in the present work.

\subsection{Purpose of the Present Paper}

These introductory remarks show, that a six dimensional yield locus based on back-extrapolated yield stresses can be conceived. It describes the basic stress levels required for all possible uniaxial or multiaxial plastic deformation modes beyond the elasto-plastic transition region. It does not describe effects related to work hardening. Such yield locus, when known, would nevertheless be a quite useful tool when analyzing complex forming operations, e.g. in FEM studies. It is however quite difficult to obtain for a given material. Von Mises or Hill yield surfaces are often used during the analysis of forming processes. These approximations are often not very satisfactory for engineering materials, which have a crystallographic texture and correspondingly an anisotropic yield locus. It is the purpose of this paper to show how such yield loci can be estimated from texture data, and how they can be used in applications such as FEM calculations of plastic forming operations.

\section{YIELD LOCI OF ANISOTROPIC MATERIALS}

\subsection{Isotropy vs. Anisotropy}

The properties of isotropic materials do not change when an arbitrary rotation is applied to the material, or conversely, when such rotation is applied to the 


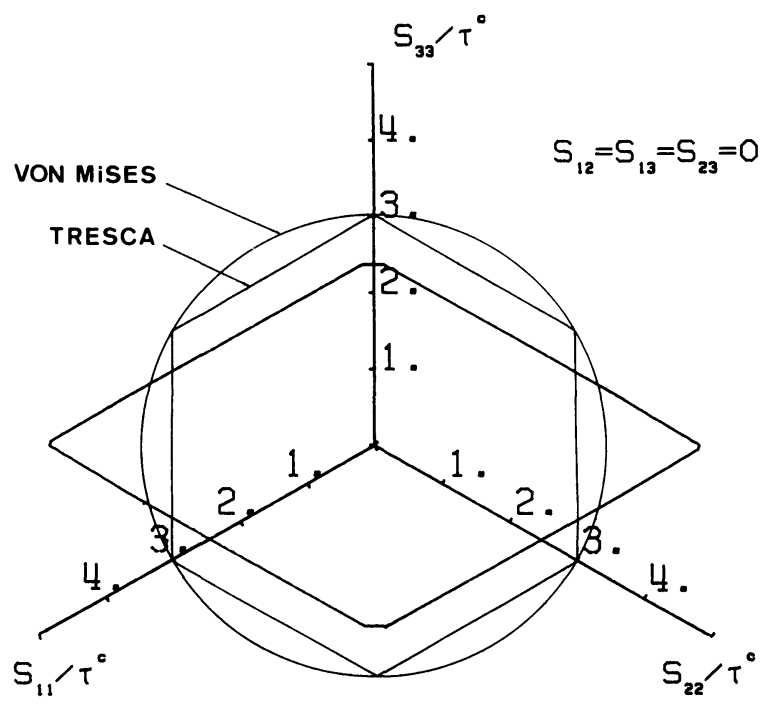

Figure $3 \pi$-plane section of the yield locus of:- the von Mises yield locus for isotropic materials; the Tresca yieid locus for isotropic materials; - a f.c.c. single crystal with a (001) [110] orientation. This yield locus has been obtained using the "geometrical method" described in Section 2.5.

reference system with respect to which the properties are expressed. Thus the shape of the yield locus must be independent of the choice of the reference system in physical space. Hence one may always choose the principal directions of the acting stress as reference system. The three principal stresses then are the only stress components that are not zero, hence a three-dimensional stress space is sufficient for the representation of the yield locus. Figure 3 shows a $\pi$-plane section $\dagger$ of the von Mises and the Tresca yield loci for isotropic materials. It is worthwhile to note that the von Mises yield condition can easily be expressed as a function of the deviatoric stresses without using the reference system of the principal stresses:

$$
F\left(\mathbf{S}^{\prime}\right)=\left(\frac{3}{2} \mathbf{S}^{\prime}: \mathbf{S}^{\prime}\right)^{1 / 2}-S_{0}=0
$$

in which $S_{0}$ is the yield stress in a uniaxial tensile test.

The reduction from six stress components to three principal stress components is not possible for anisotropic materials, since the yield locus would not be independent of the direction of the principal stresses. This has the obvious disadvantage that one really needs six dimensions in stress space for a full representation of the yield locus. $\ddagger$ It has the advantage that one does not need to adapt the reference system in physical space to the principal directions of the acting stress.

For many applications (e.g. for sheet forming, with $x_{3}$ being the normal to the sheet), one may assume that $S_{23}=S_{31}=0$. This then reduces the number of

$\dagger$ A section by the plane $S_{11}+S_{22}+S_{33}=0$.

$\ddagger$ For plastically incompressible materials, one can reduce the number of independent stress components to five, see e.g. Lequeu et al. (1987) or Van Houtte (1988). Only the deviatoric component of the stress (represented by $\mathbf{S}^{\prime}$ ) is relevant in such case. This will be assumed throughout this paper. 
dimensions to be considered to four, and may furthermore greatly simplify the analysis (Van Houtte, 1987). This simplification cannot be made for threedimensional studies of processes such as forging.

\subsection{Polycrystalline Materials}

Engineering materials usually consist of many tiny crystallites. The plastic deformation of these is achieved by slip on particular slip systems, i.e. on particular crystallographic planes and in particular directions. As a result, the yield locus of such crystallite is strongly anisotropic and may differ very much from the well-known shape of the von Mises or the Tresca yield surface (Figure 3).

This anisotropy may be cancelled out completely if in a polycrystal all crystal orientations are equally represented. In that case, the orientation distribution function $f(g)$ that describes the texture (Bunge, 1982) is equal to 1 for all crystal orientations $g$. So the material will have an isotropic yield locus, but not necessarily the "classical" von Mises-yield locus nor the Tresca-yield locus.

However, for most engineering materials $f(g)$ is not constant but depends on $g$. As a result, the macroscopic yield locus will be anisotropic as well, but usually not to the same extent as the single crystal yield locus.

\subsection{Calculation of Yield Loci}

A method is needed that finds all possible macroscopic stresses $\mathbf{S}^{\prime}$ that may start plastic deformation in a given material. Mechanical tests can give some information, but are very time consuming and can in practice never lead to a complete six-dimensional yield locus. So theoretical models are preferred. From the outside, such models may operate in two ways:

(i) The plastic strain rate tensor $\dot{\mathbf{E}}$ is prescribed; the model calculates the required stress $\mathbf{S}^{\prime}$. This calculation is repeated for a sufficient number of different tensors $\dot{\mathbf{E}}$ to be able to estimate the full $\boldsymbol{F}\left(\mathbf{S}^{\prime}\right)$ surface.

(ii) The (deviatoric) stress mode $\mathbf{U}^{\prime}$ is chosen. The model calculates the yield stress $\mathbf{S}^{\prime}$ that corresponds to it (Figure 1) as well as the strain mode given by Eq. (3). The calculation is repeated a sufficient number of times for different stress modes.

Mixed boundary conditions may in principle also be chosen.

Which strategy is to be chosen depends on the possibilities of the model that one wants to use. Most authors prefer to use strategy (i). We will limit this discussion to that case.

So a macroscopic plastic strain $\dot{\mathbf{E}}$ is chosen. This information then serves as input for a model for the plastic deformation of a polycrystal, which must take the microscopic deformation mechanisms of each crystal into account. Such model is faced with several questions:

(i) how are the microscopic stresses $\sigma$ and strain rates $\dot{\varepsilon}$ distributed throughout the polycrystal?

(ii) what is the value of the macroscopic stress $\mathbf{S}^{\prime}$ ? 
A simplification that is almost always made is, that the microscopic stresses and strain rates that are computed or assumed are uniformly distributed within a particular cyrstallite. Such is the case for the so-called self-consistent models that set up a comprehensive set of equations for each grain (e.g. Berveiller and Zaoui, 1984; Molinari, Canova and Ahzi, 1987). The strain rates of each grain may be different from each other, but their average must be equal to the macroscopic strain rate. At a given moment, the microscopic strain rate of a given grain will depend on the capability of the matrix surrounding the grain to exercise the stress required to impose that strain rate on the grain. Each of these models has its own assumptions for the calculation of this interaction grain-matrix. The matrix is assumed to be a continuous solid that can absorb a inhomogeneous deformation. Some of these models are elastic-plastic, others neglect the elastic part of the deformation. Self-consistent models can be used to calculate yield loci (Berveiller and Zaoui, 1984).

The Taylor-Bishop-Hill theory (see e.g. Van Houtte, 1988) makes even stronger assumptions: it assumes the microscopic strain rate $\dot{\varepsilon}$ to be homogeneous throughout the whole polycrystal and hence to be equal to the macroscopic strain rate $\mathbf{E}$. The microscopic plastic stresses are calculated for each crystallite and their equilibrium at the grain boundaries is even less satisfied than by the self-consistent models. On the other hand, there are no incompatibilities in the distribution of the microscopic strains throughout the polycrystal. The TaylorBishop-Hill theory will be adopted in the rest of this paper.

\subsection{Elaboration Using the Series Expansion Method and the Taylor-Bishop-Hill Theory}

The Taylor-Bishop-Hill theory assumes the microscopic strain rate at a given time to be equal to the macroscopic strain rate:

$$
\dot{\boldsymbol{\varepsilon}}=\dot{\mathbf{E}}
$$

The microscopic strain rate is then used to find the activated slip systems of a grain with a known orientation $g$ and from these the local microscopic plastic stress $\sigma$ (see e.g. Van Houtte, 1988). So $\sigma$ is a function of $g$ and of $\left(\dot{\mathbf{E}} / E_{m}\right)$, which stands for the strain mode. The scalar $\dot{E}_{m}$ is a macroscopic measure of the strain rate, such as the von Mises effective strain rate (see for example Aernoudt et al., 1987). Strain rate sensitivity is neglected in the present analysis; so the stress $\sigma$ is independent from the level of the strain rate. It only depends on the strain mode.

It can be demonstrated that for a single-phase material, under the assumptions of the Taylor-Bishop-Hill theory, the average of all microscopic stresses must be equal to the macroscopic stress:

$$
\mathbf{S}\left(\dot{\mathbf{E}} / \dot{E}_{m}\right)=\int \boldsymbol{\sigma}\left(g, \dot{\mathbf{E}} / \dot{E}_{m}\right) f(g) \mathrm{d} g
$$

in which $f(g)$ is the orientation distribution function (O.D.F.) that describes the texture of the material (Bunge, 1982).

Equation (11) is not very convenient in practical calculation. Most authors prefer to base their analysis on the power dissipation per unit volume. The 
microscopic plastic power dissipation per unit volume is given by

$$
P\left(g, \dot{\mathbf{E}} / \dot{E}_{m}\right)=\dot{E}_{m} \tau^{c} M\left(g, \dot{\mathbf{E}} / \dot{E}_{m}\right)
$$

$M\left(g, \dot{\mathbf{E}} / \dot{E}_{m}\right)$ is the Taylor factor for the considered orientation and strain mode. It is a well-known result of a Taylor-Bishop-Hill calculation (Van Houtte, 1988). $\tau^{c}$ is the critical resolved shear stress.

It is very convenient in this analysis to express the Taylor factors as series expansions of harmonic functions (Bunge, 1970, 1982):

$$
M\left(g, \dot{\mathbf{E}} / \dot{E}_{m}\right)=\sum_{\ell} \sum_{\mu} \sum_{v} M_{\ell}^{\mu v}\left(\dot{\mathbf{E}} / \dot{E}_{m}\right) \dot{T}_{\ell}^{\mu v}(g)
$$

The series expansion coefficients $M_{\ell}^{\mu \nu}\left(\dot{\mathbf{E}} / \dot{E}_{m}\right)$ are obtained by integrating the Taylor factors through Euler space:

$$
M_{\ell}^{\mu v}\left(\dot{\mathbf{E}} / \dot{E}_{m}\right)=(2 l+1) \int M\left(g, \dot{\mathbf{E}} / \dot{E}_{m}\right) \dot{T}_{l}^{\mu v}(g) \mathrm{d} g
$$

The Taylor factors themselves must be calculated by the Taylor-Bishop-Hill model for a sufficient number of orientations $g$ and for a sufficient number of strain modes $\dot{\mathbf{E}} / \dot{E}_{m}$. This work can be organized in an efficient way as has been explained by several authors (Bunge, 1970; Bunge, Schulze and Greszik, 1980; Sowerby, Da Viana and Davies, 1980). The methods proposed by these authors produce "principal strain yield loci," i.e. $x-y$ plots that do not really represent plane sections of a yield locus. Suppose that $\mathbf{S}_{x}$ in Eq. (2) represents a uniaxial stress in a direction that makes an angle $\alpha$ to the rolling direction of a sheet, and $\mathbf{S}_{y}$ in a direction $(\alpha+\pi / 2)$. These directions are principal directions of stress for all stress states of the section to be considered. The above authors however also assume that these directions are principal directions of the strain rate. But the directions $\alpha$ and $(\alpha+\pi / 2)$ can only simultaneously be principal directions of stress and strain if they also are twofold rotational symmetry axes of the "sample symmetry" of the sample's texture. Such condition is in general only satisfied for $\alpha=0$ and $\alpha=\pi / 2$, not for other directions. Mols, Van Praet and Van Houtte (1984) and Van Houtte (1987) proposed feasible strategies for the choice of the set of strain modes to be considered that avoid to make the assumption that the principal directions of stress and strain rate coincide. However, Van Houtte (1987) still accepted this simplification for the axis $x_{3}$ (the normal to the sheet), which strongly reduces the complexity of the calculations.

Whatever the method used, the $M_{\ell}^{\mu v}\left(\dot{\mathbf{E}} / \dot{E}_{m}\right)$ can be calculated once and for all (for a given metal) and stored for later use. The calculation of the yield loci of samples with different textures then becomes a quite short calculation. It is based on the calculation of the average plastic power dissipation throughout the polycrystal:

$$
P_{m}\left(\dot{\mathbf{E}} / \dot{E}_{m}\right)=\dot{E}_{m} \tau^{c} M_{m}\left(\dot{\mathbf{E}} / \dot{E}_{m}\right)
$$

in which $M_{m}\left(\dot{\mathbf{E}} / \dot{E}_{m}\right)$ is the average Taylor factor of the sample. It is given by:

$$
M_{m}\left(\dot{\mathbf{E}} / \dot{E}_{m}\right)=\int M\left(g, \dot{\mathbf{E}} / \dot{E}_{m}\right) f(g) \mathrm{d} g
$$

The integral can be elaborated using Eq. (13) and the properties of the series 
expansion:

$$
M_{m}\left(\dot{\mathbf{E}} / \dot{E}_{m}\right)=\sum_{\ell} \sum_{\mu} \sum_{v} M_{\ell}^{\mu v}\left(\dot{\mathbf{E}} / \dot{E}_{m}\right) C_{\ell}^{\mu v} /(2 l+1)
$$

in which the $C_{\ell}^{\mu \nu}$ are the $C$-coefficients of the O.D.F. A modern computer can evaluate $M_{m}$ for thousands of strain modes per second using this expression.

Equation (15) is not the only expression for the average plastic power dissipation per unit volume. It is also equal to

$$
P_{m}\left(\dot{\mathbf{E}} / \dot{E}_{m}\right)=\mathbf{S}: \dot{\mathbf{E}}=S_{i j} \dot{E}_{i j}
$$

in which $\mathbf{S}$ is the macroscopic stress required to achieve the plastic strain mode $\dot{\mathbf{E}} / \dot{E}_{m}$. Combining eqs. (15) and (18) leads to

$$
\left(S_{i j} / \tau^{c}\right)\left(\dot{E}_{i j} / \dot{E}_{m}\right)=M_{m}\left(\dot{\mathbf{E}} / \dot{E}_{m}\right)
$$

In stress space, this represents a hyperplane that contains the plastic stress $\mathbf{S}$. There is such hyperplane for each strain mode. A large number of such hyperplanes (one for each strain mode $\dot{\mathbf{E}} / \dot{E}_{m}$ ) is easily obtained for any sample with a known texture, using for example the strategies proposed by Mols et al. (1984) or by Van Houtte (1987). These strategies consider all possible strain modes within an angular resolution of $10^{\circ}$, respectively in six dimensional stress-strain space or in four dimensional stress-strain space (assuming the existence of a diad axis of the texture at $x_{3}$, and assuming applications for which $S_{23}=S_{31}=0$ ). This set of hyperplanes contains in principle the description of the yield locus, but not in a convenient form. It will be explained in the next sections how this set of hyperplanes can be used for making a graphical representation of the yield locus, or for using it in engineering applications.

\subsection{Stress Level for a given Stress Mode Plane Sections of the Yield Locus}

For many applications, including the graphical representations of plane sections of the yield locus, it is required to find out what the stress level $S_{Y}$ is for a given direction $U$ in stress space, as shown by Figure 1 and by Eq. (4). From Eq. (5) it follows that for any stress in the direction $\mathbf{U}$

$$
S_{i j}=S U_{i j}
$$

From Eqs. (19-20), it follows that

$$
S\left(\dot{\mathbf{E}} / \dot{E}_{m}\right)=\frac{\tau^{c} M_{m}\left(\dot{\mathbf{E}} / \dot{E}_{m}\right)}{U_{i j} \dot{E}_{i j} / \dot{E}_{m}}=\frac{\tau^{c} M_{m}\left(\dot{\mathbf{E}} / \dot{E}_{m}\right)}{\mathbf{U}:\left(\dot{\mathbf{E}} / \dot{E}_{m}\right)}
$$

$S\left(\dot{\mathbf{E}} / \dot{E}_{m}\right)$ is a measure for the distance between the origin of stress-strain space and the intersection of the direction $U$ with the hyperplane associated to the strain mode $\dot{\mathbf{E}} / \dot{E}_{m}$. The plastic stress $S_{y}$ is found for that hyperplane for which $S$ is positive but otherwise minimal:

$$
S_{Y}=\operatorname{Min}\left[S\left(\dot{\mathbf{E}} / \dot{E}_{m}\right)\right] \quad \text { with } \quad \mathbf{U}:\left(\dot{\mathbf{E}} / \dot{E}_{m}\right) \geq 0
$$

This principle allows to identify the hyperplane of which the intersection with the direction $\mathbf{U}$ is closest to the intersection of this hyperplane and the yield locus. Mols et al. (1984) and Van Houtte (1987) used a purely geometrical method 


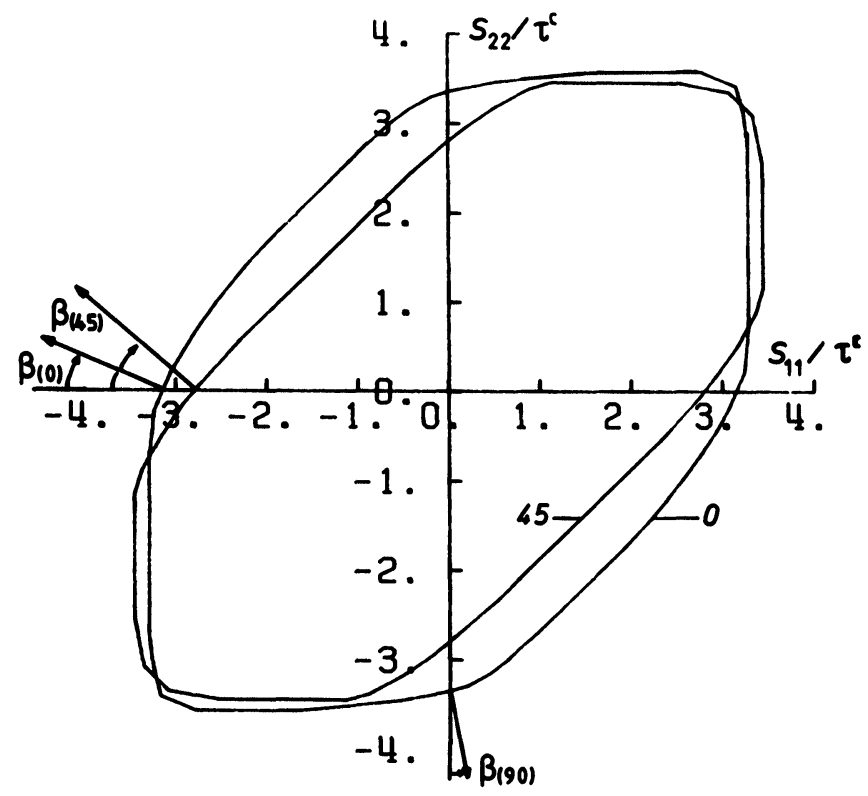

Figure 4 Two examples of $S_{11}-S_{22}$ sections of a yield locus of a cold rolled aluminium sheet, for $\alpha=0^{\circ}\left(x_{1}\right.$ is the rolling direction) and for $\alpha=45^{\circ}$ ( $x_{1}$ at $45^{\circ}$ of the rolling direction).

(without interpolation) in which the intersection with this "closest hyperplane" was used as an estimation of the intersection with the yield locus itself. The method was used to generate plane sections as those described by Eq. (2). This can be achieved by generating a series of stress modes $U$ as follows:

$$
\mathbf{U}(\theta)=\mathbf{U}_{x} \cos \theta+\mathbf{U}_{y} \sin \theta
$$

$\mathbf{U}_{x}$ and $\mathbf{U}_{y}$ are two non-parallel stress modes. $\dagger \theta$ is varied in small steps, and for each resulting stress mode, $S_{y}$ is calculated using Eqs. (21-22). Figure 3 is an example of a result of this procedure for a nearly-single crystal of a f.c.c. metal with $\{111\}\langle 110\rangle$ slip systems. Figure 4 shows an example for a cold rolled aluminium sample. The advantage of the purely geometrical method is, that it can reproduce the yield loci of single crystals including the sharp corners. The drawback is, that such geometrical representation can hardly be used for sophisticated engineering applications such as finite element calculations. For this purpose, we are developing an analytical method for the representation of yield loci, which basically is a six dimensional interpolation method based on the discrete set of average Taylor factors of Eq. (17).

The geometrical method proposed by Van Houtte (1987) is implemented in Van Houtte's "MTM-QTA" O.D.F.-software package $\ddagger$ for cubic metals. It assumes that $x_{3}$ is a diad axis of the sample texture, which is nearly always the case for sheet materials, and that the stress components $S_{23}$ and $S_{31}$ are zero.

$\dagger \mathbf{U}_{x}$ may for example represent a uniaxial stress in a direction of a rolled sheet that makes an angle $\alpha$ with the rolling direction, and $\mathbf{U}_{y}$ at a direction $(\alpha+\pi / 2)$.

$\ddagger$ For IBM-compatible personal computers. 


\subsection{Analytical Representation of a Yield Locus}

A well-known example of Eq. (1) is Hill's yield locus, which basically is a quadratic expression:

$$
A_{i j k l} S_{i j} S_{k l}=B
$$

In the classical expression for Hill's yield locus, Eq. (24) is simplified by assuming that the coordinate axes are diad axes. It can be shown that many $A_{i j k l}$ coefficients must be zero because of this. An additional condition arises from the fact, that it is assumed that the yield condition is independent from the hydrostatic part of the stress.

Equation (24) is insufficient for a proper description of the yield locus of most anisotropic materials. Its approximation of the yield locus of the (001)[110] crystal shown by Figure 3 would for example be an ellipse. One may of course generalize Eq. (24) and add third or fourth order terms to it. We have however decided to follow a different way because of the requirements of finite element methods. The algorithms of these methods require at some stage to calculate the plastic stress tensor from a given plastic strain rate tensor. So $\dot{\mathbf{E}}$ must be the independent variable, and $\mathbf{S}$ the result. In Eqs. such as (1), (24) and (3) it is of course the other way around, and it is in general not easy to invert the expressions.

In order to overcome this difficulty, a plastic potential of "type II" is proposed here. It is a sort of plastic potential expressed as a function of the strain mode, and from which the plastic stress (relative to the critical resolved shear stress) can be derived.

In this analysis, it will be assumed that the material is incompressible and that only the deviatoric stress matters with regard to plastic deformation. For the sake of simplicity, all deviatoric stress tensors $\mathbf{S}^{\prime}$ and strain rate tensors $\dot{\mathbf{E}}$ are converted into five-dimensional stress vectors $\mathbf{s}$ and strain rate vectors $\dot{\mathbf{e}}$ using the method proposed by Van Houtte (1988). It is reminded here that this vector representation has the following properties:

$$
v_{p} \times w_{p}=\mathbf{v} \cdot \mathbf{w}=\mathbf{V}: \mathbf{W}=V_{i j} W_{i j}
$$

in which $\mathbf{v}$ and $\mathbf{w}$ are two vectors (stress, or strain rate, or mixed) and $\mathbf{V}$ and $\mathbf{W}$ the corresponding tensors. $p$ and $q$ are indices that run from 1 to 5 . For the macroscopic measure of the strain rate $\dot{E}_{m}=\dot{e}_{m}$, the von Mises equivalent strain rate is used:

$$
\dot{e}_{m}=\dot{E}_{m}=\left(\frac{2}{3} \dot{e}_{p} \dot{e}_{p}\right)^{1 / 2}
$$

$\dot{e}_{m}$ can be seen as a function of $\dot{e}_{p}$. II:

The following function of the five $\dot{e}_{p}$ can be seen as a plastic potential of type

$$
Q\left(\dot{e}_{p}\right)=\dot{e}_{m}\left(\dot{e}_{p}\right) G\left(\dot{e}_{p} / \dot{e}_{m}\right)
$$

By definition, the stresses are derived from $Q$ as follows:

$$
\frac{s_{p}}{\tau^{c}}=\frac{\partial Q\left(\dot{e}_{p}\right)}{\partial \dot{e}_{p}}=\frac{2}{3}\left(\frac{\dot{e}_{p}}{\dot{e}_{m}}\right) G+\frac{\partial G}{\partial\left(\dot{e}_{q} / \dot{e}_{m}\right)}\left[\delta_{p q}-\frac{2}{3}\left(\frac{\dot{e}_{p}}{\dot{e}_{m}}\right)\left(\frac{\dot{e}_{q}}{\dot{e}_{m}}\right)\right]
$$

which clearly is an expression depending only on the ratios $\left(\dot{e}_{p} / \dot{e}_{m}\right)$, i.e. on the strain mode. So the type II plastic potential leads to $s_{p} / \tau^{c}$ ratios that are strain 
rate independent; if necessary, strain rate sensitivity can be brought in by making $\tau^{c}$ a function of $\dot{e}_{m}$.

The function $Q\left(\dot{e}_{p}\right)$ as derived above has a very interesting property:

$$
Q=\dot{e}_{p} \frac{\partial Q}{\partial \dot{e}_{p}}
$$

which can be demonstrated by substituting $Q$ and its partial derivatives by their expressions Eq. (27) and Eq. (28). It then follows from Eqs. (28) and (29) that

$$
Q=\dot{e}_{p}\left(s_{p} / \tau^{c}\right)
$$

This can be used to demonstrate that the plastic stresses and strain rates obtained in this way indeed satisfy the normality rule (Eq. (3)). Basically Eqs. (27-28) are a parametric representation of the plastic stress states $\mathbf{s}$. The strain rates $\dot{\mathbf{e}}$ are the parameters. A change de of the parameters causes a change ds of the stress. The scalar product $\dot{\mathbf{e}} \cdot \mathbf{d s}$ must be zero if the vector $\dot{\mathbf{e}}$ (which represents the strain rate tensor) is to be normal to the yield locus. This condition can be written as follows:

$$
\dot{e}_{p} \mathrm{~d} s_{p}=\dot{e}_{p} \frac{\partial s_{p}}{\partial \dot{e}_{q}} \mathrm{~d} \dot{e}_{q}=0
$$

Now let us calculate the expression

$$
\mathrm{d} Q=\frac{\partial Q}{\partial \dot{e}_{q}} \mathrm{~d} \dot{e}_{q}
$$

in two ways, first using Eq. (30):

$$
\mathrm{d} Q=\left(\delta_{p q} s_{p} \mathrm{~d} \dot{e}_{q}+\dot{e}_{p} \frac{\partial s_{p}}{\partial \dot{e}_{q}} \mathrm{~d} \dot{e}_{q}\right) / \tau^{c}=\left(s_{q} \mathrm{~d} \dot{e}_{q}+\dot{e}_{p} \mathrm{~d} s_{p}\right) / \tau^{c}
$$

and secondly, using Eq. (28):

$$
\mathrm{d} Q=\left(s_{q} / \tau^{c}\right) \mathrm{d} \dot{e}_{q}
$$

Eqs. (33) and (34) are both true. This is only possible when $\dot{e}_{p} \mathrm{~d} s_{p}$ is zero. So Eq. (31) is satisfied.

Comparison of Eq. (30) to Eq. (18) (and taking account of Eq. (25)) shows that $Q$ is equal to $P_{m} / \tau^{c}$. It then follows from Eq. (15) and Eq. (27) that $G\left(\dot{e}_{p} / \dot{e}_{m}\right)$ is nothing else than $M_{m}\left(\dot{\mathbf{E}} / \dot{E}_{m}\right)$, the average Taylor factor for the strain mode $\dot{\mathbf{E}} / \dot{E}_{m}$. Hence it is possible to calculate the values of $G$ for all possible strain modes from the texture, as explained in the previous sections. An analytical expression for $G$ is then fitted to these data. In a first study, we have used the following expression:

$$
G\left(\dot{e}_{p} / \dot{e}_{m}\right)=\frac{a_{p q} \dot{e}_{p} \dot{e}_{q}}{\dot{e}_{m}^{2}}+\frac{b_{p q r s} \dot{e}_{p} \dot{e}_{q} \dot{e}_{r} \dot{e}_{s}}{\dot{e}_{m}^{4}}+\frac{c_{p q r s t u} \dot{e}_{p} \dot{e}_{q} \dot{e}_{r} \dot{e}_{s} \dot{e}_{t} \dot{e}_{u}}{\dot{e}_{m}^{6}}
$$

i.e., a general sixth order expression containing only terms of even rank. This means that the yield locus must be centrosymmetric. There is a considerable number of coefficients such as the $c_{\text {pqrstu }}$. However, many of these are always zero because of various symmetry properties of this "tensor." The feasibility of this 
method depends on one's ability to identify these zero coefficients and on one's programming skills.

Once the coefficients of the expression (35) have been found by least squares fitting to the average Taylor factors, analytical expressions can be derived for the stresses using Eq. (28). These then allow high-speed calculations of the stress components from the strain rates, for application in the F.E.M. algorithms.

It is of course also possible to use Eqs. (21-23) (in which $M_{m}$ is replaced by $G$ ) in order to produce plane sections of the yield locus represented by Eq. (35). The minimum of Eq. (22) can be found by means of a pseudo-Newtonian iteration.

Two examples of results will be shown here for f.c.s. metals with $\{111\}\langle 110\rangle$ slip systems:

(i) for a rather sharp texture, i.e. a (001) [110] orientation with a Gaussian distribution around it:

$$
f(g)=f_{0} e^{-\left(\Phi / \Phi_{0}\right)^{2}}
$$

$\Phi$ is the angular distance from (001) [110] to $g . \Phi_{0}$ was given the value $16.5^{\circ} . f_{0}$ is adjusted in order to normalize the function (Bunge, 1982).

(ii) for a texture taken from a cold rolled 3004 aluminium alloy.

Example for the sharp texture. Figure 5 is a polar diagram that shows the analytical approximation of the function $G\left(\dot{e} / \dot{e}_{m}\right)$ by means of Eq. (31) in the $\pi$-plane of stress-strain space. $\dot{\mathbf{e}} / \dot{e}_{m}$ is unit vector in stress-strain space, so it basically describes a direction. It is reminded here that $G\left(\dot{\mathbf{e}} / \dot{e}_{m}\right)$ is nothing else than the average Taylor factor $M_{m}\left(\dot{\mathbf{E}} / \dot{E}_{m}\right)$. The function shown by Figure 5 has then been used to derive the $\pi$-plane section of the yield locus (Figure 6a). Figure

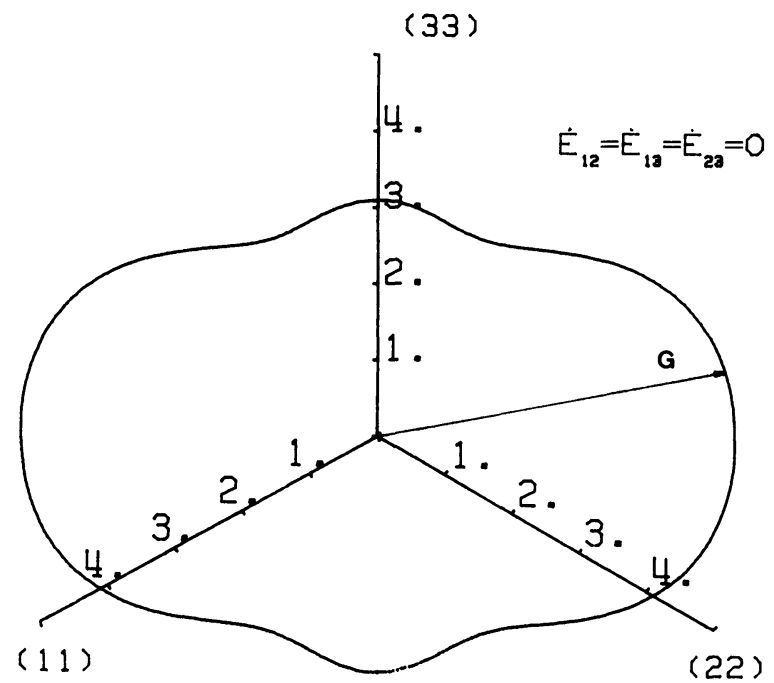

Figure 5 An analytical approximation of the function $G\left(\dot{\mathbf{e}} / \dot{e}_{m}\right)$ (i.e., the average Taylor factor as a function of the strain mode) for a f.c.c. metal with a moderately sharp texture: a Gaussian distribution (Eq. 36) around the orientation (001) [110]. The drawing is a polar diagram in the $\pi$-plane section of stress-strain space. (The strain mode is represented by a direction in this space). 
6b shows the same yield locus section, this time obtained by the geometrical method described in section 2.5. Figure 3 shows how this section would look like for a single crystal. It must be emphasized here that it is difficult for the analytical method to represent sharp corners of yield loci. It is not capable of representing the yield loci of much sharper textures than the one of this example. The purely

(a)

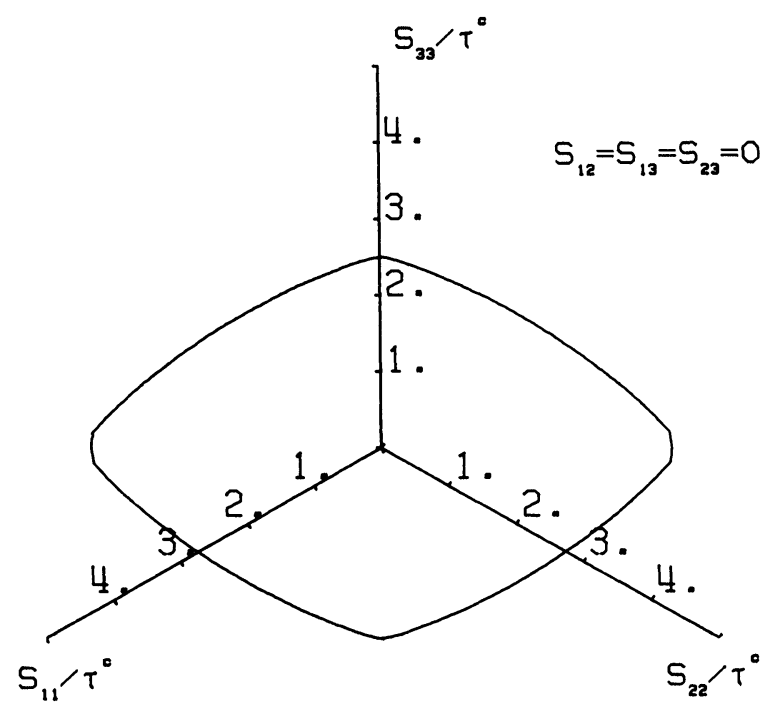

(b)

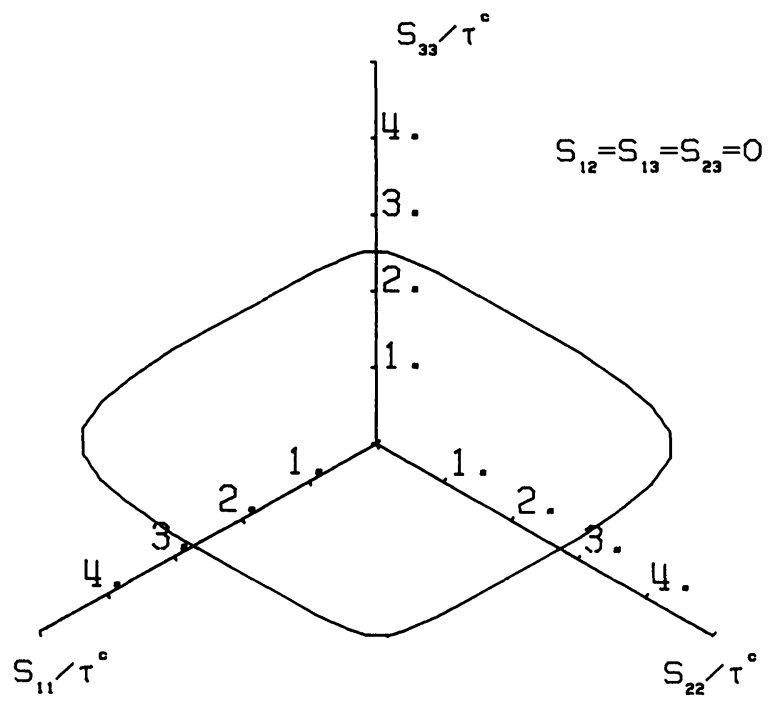

Figure 6 (a) $\pi$-plane section of the yield locus of a f.c.c. metal with a moderately sharp texture: a Gaussian distribution (Eq. (36)) around the orientation (001) [110] (analytical method). (b) Idem, geometrical method. 


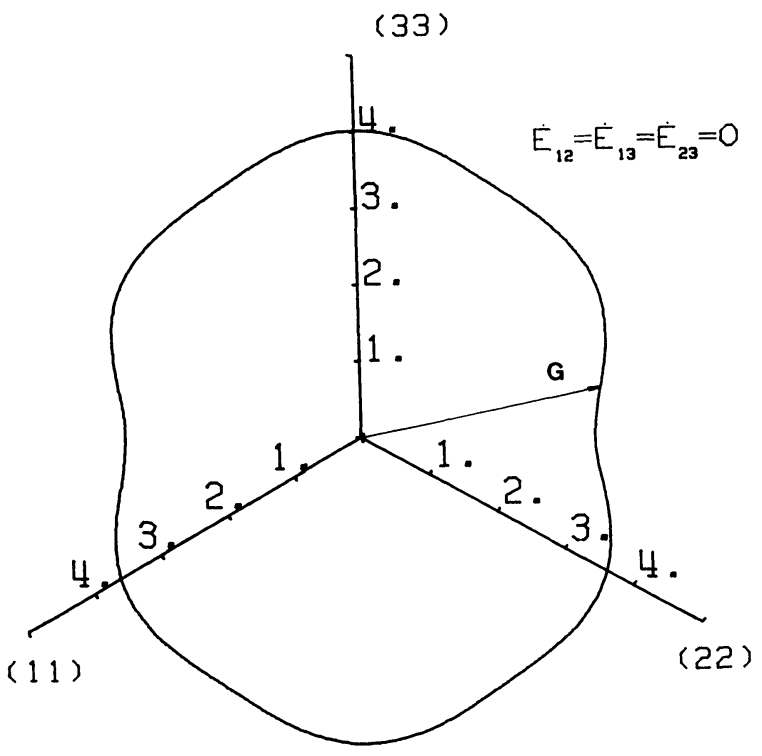

Figure 7 An analytical approximation of the function $G\left(\dot{\mathbf{e}} / \dot{e}_{m}\right)$ (i.e., the average Taylor factor as a function of the strain mode) for a cold rolled 3004 aluminium alloy. The drawing is a polar diagram in the $\pi$-plane section of strain space. (The strain mode is represented by a direction in this space). Strains defined for $\alpha=45^{\circ}$ ( $x_{1}$ at $45^{\circ}$ with the rolling direction).

geometrical method described in section 2.5 is much more powerful in this regard, as is demonstrated by the example shown by Figure 3.

Example for a cold rolled 30004-alloy. Figure 7 shows the polar diagram in the $\pi$-plane of the function $G\left(\dot{\mathbf{e}} / \dot{e}_{m}\right)$. Figure 8 a shows the yield locus derived from it. The representation of yield loci of such industrial materials by the analytical method is usually unproblematic. For comparison, Figure $8 \mathrm{~b}$ shows the same section produced by the geometrical method.

\section{SUMMARY AND CONCLUSIONS}

The series expansion method for the representation of orientation distribution functions makes it possible to obtain the yield surface of a textures sample in full six dimensional stress space. No simplifying assumptions such as coincidence of principal directions of stresses and strains are necessary. The calculations make use of pre-calculated Taylor factors for a variety of strain modes. The method could also be based on the results of other models for the plastic deformation of polycrystals than the Taylor theory.

A method is proposed for an analytical representation of such six dimensional yield loci. It will allow to replace von Mises' yield criterion by a yield criterion based on the actual yield locus in elasto-plastic finite element calculations of forming processes. It has been found that this method works well for weak and moderately sharp textures, but not for extremely sharp textures. 
(a)

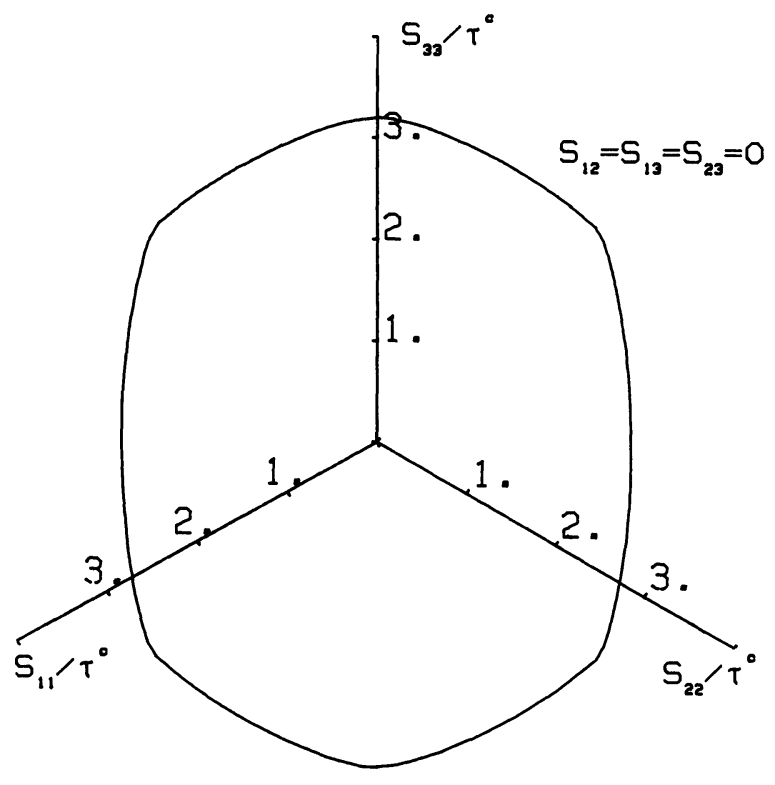

(b)

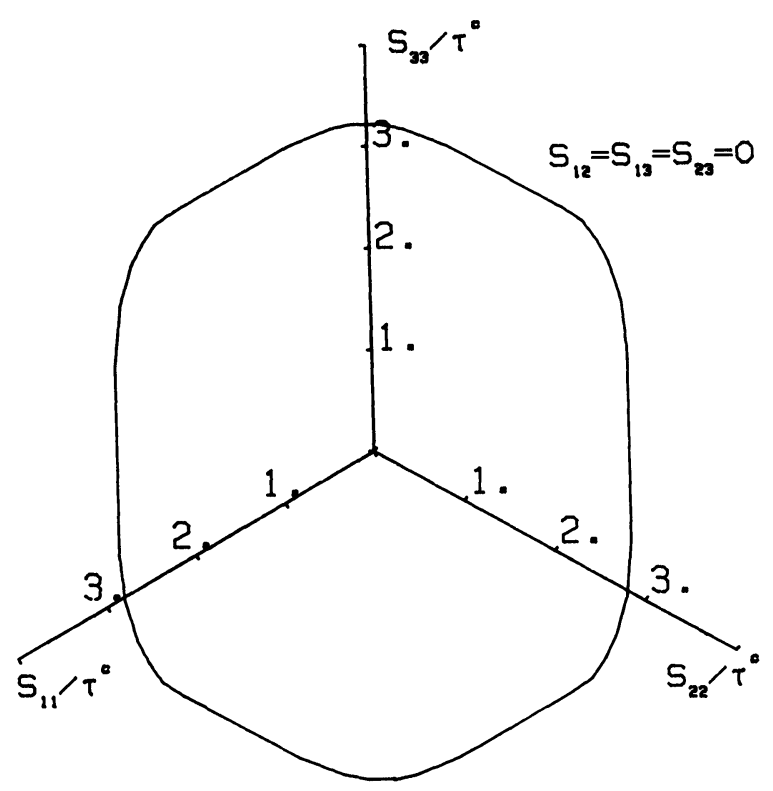

Figure 8 (a) $\pi$-plane section of the yield locus of a cold rolled 3004 aluminium alloy (analytical method). Stresses defined for $\alpha=45^{\circ}$ ( $x_{1}$ at $45^{\circ}$ with the rolling direction). (b) Idem, geometrical method. 


\section{References}

Aernoudt, E., Gil-Sevillano, J. and Van Houtte, P. (1987). Constitutive Relations and Their Physical Basis (Proc. 8th Risö International Symposium on Metallurgy and Materials Science), pp. 1-38, S. I. Andersen, J. B. Bilde-Sörensen, N. Hansen, T. Leffers, H. Lilholt, O. B. Pedersen and B. Ralph, eds., Risö National Laboratory, Roskilde, Denmark.

Berveiller, M. and Zaoui, A. (1984). J. Engng. Mater. Technol. 106, 295-298.

Bunge, H. J. (1970). Kristall und Technik 5, 145-175.

Bunge, H. J. (1982). Texture Analysis in Material Science, Butterworths, London.

Bunge, H. J., Schulze, M. and Grzesik, D. (1980). Peine-Salzgitter Berichte, Sonderheft.

Hill, R. (1950). The Mathematical Theory of Plasticity, Clarendon Press, Oxford.

Lequeu, Ph., Gilormini, P., Montheillet, F., Bacroix, B. and Jonas, J. J. (1987). Acta Metall. 35, 439-451.

Molinari, A., Canova, G. R. and Ahzi, S. (1987). Acta Metall. 35, 2983-2994.

Mols, K., Van Praet, K. and Van Houtte, P. (1984). Proc. Seventh Intntl. Conf. on Textures of Materials (ICOTOM 7), pp. 651-656, C. M. Brakman, P. Jongenburger and E. J. Mittemeijer, eds., Netherlands Society for Material Science, Zwijndrecht (The Netherlands).

Sowerby, R., Da Viana, C. S. and Davies, G. J. (1980). Mat. Sci. Eng. 46, 23-51.

Van Houtte, P. (1987). Textures and Microstructures 7, 29-72.

Van Houtte, P. (1988). Textures and Microstructures, 8-9, 313-350. 